\title{
Integral operators on new families of meromorphic functions of complex order
}

\author{
Aabed Mohammed and Maslina Darus ${ }^{*}$
}

* Correspondence: maslina@ukm. my

School of Mathematical Sciences, Faculty of Science and Technology, Universiti Kebangsaan Malaysia, 43600 Bangi, Selangor Darul Ehsan, Malaysia

\section{Abstract}

In this article, we define and investigate new families of certain subclasses of meromorphic functions of complex order. Considering the new subclasses, several properties for certain integral operators are derived.

2010 Mathematics Subject Classification: 30C45.

Keywords: Analytic function, meromorphic function, integral operator

\section{Introduction}

Let $\Sigma$ denote the class of functions of the form:

$$
f(z)=\frac{1}{z}+\sum_{n=0}^{\infty} a_{n} z^{n},
$$

which are analytic in the punctured open unit disk

$$
\mathbb{U}^{*}=\{z \in \mathbb{C}: 0<|z|<1\}=\mathbb{U} \backslash\{0\},
$$

where $\mathbb{U}$ is the open unit disk $\mathbb{U}=\{z \in \mathbb{C}:|z|<1\}$.

We say that a function $f \in \Sigma$ is meromorphic starlike of order $\delta(0 \leq \delta<1)$, and belongs to the class $\Sigma^{*}(\delta)$, if it satisfies the inequality:

$$
-\Re\left(\frac{z f^{\prime}(z)}{f(z)}\right)>\delta .
$$

A function $f \in \Sigma$ is a meromorphic convex function of order $\delta(0 \leq \delta<1)$, if $f$ satisfies the following inequality:

$$
-\Re\left(1+\frac{z f^{\prime \prime}(z)}{f^{\prime}(z)}\right)>\delta,
$$

and we denote this class by $\Sigma_{k}(\delta)$.

For $f \in \Sigma$, Wang et al. [1] and Nehari and Netanyahu [2] introduced and studied the subclass $\Sigma_{N}(\beta)$ of $\Sigma$ consisting of functions $f(z)$ satisfying

$$
-\Re\left(\frac{z f^{\prime \prime}(z)}{f^{\prime}(z)}+1\right)<\beta \quad(\beta>1, z \in \mathbb{U}) .
$$


Let $\mathcal{A}$ denote the class of functions $f$ of the form $f(z)=z+\sum_{n=2}^{\infty} a_{n} z^{n}$, which are analytic in the open unit disk $\mathbb{U}$.

Analogous to several subclasses [3-10] of analytic functions of $\mathcal{A}$, we define the following subclasses of $\Sigma$.

Definition 1.1 Let a function $f \in \Sigma$ be analytic in $U^{*}$. Then $f$ is in the class $\Sigma_{b}^{\star}(\delta)$ if, and only if, $f$ satisfies

$$
\Re\left\{1-\frac{1}{b}\left(\frac{z f^{\prime}(z)}{f(z)}+1\right)\right\}>\delta,
$$

where $b \in \mathbb{C} \backslash\{0\}, 0 \leq \delta<1$.

Definition 1.2 Let a function $f \in \Sigma$ be analytic in $\mathbb{U}^{*}$. Then $f$ is in the class $\Sigma K_{b}(\delta)$ if, and only if, $f$ satisfies

$$
\Re\left\{1-\frac{1}{b}\left(\frac{z f^{\prime \prime}(z)}{f^{\prime}(z)}+2\right)\right\}>\delta,
$$

where $b \in C \backslash\{0\}, 0 \leq \delta<1$. We note that $f \in \Sigma K_{b}(\delta)$ if, and only if, $-z f^{\prime} \in \Sigma_{b}^{\star}(\delta)$.

Furthermore, the classes

$$
\Sigma_{1}^{\star}(\delta) \equiv \Sigma^{\star}(\delta), \quad \Sigma K_{1}(\delta) \equiv \Sigma_{k}(\delta)
$$

are the classes of meromorphic starlike functions of order $\delta$ and meromorphic convex functions of order $\delta$ in $\mathbb{U}^{*}$, respectively. Moreover, the classes

$$
\Sigma_{1}^{\star}(0) \equiv \Sigma^{\star}(0), \quad \Sigma K_{1}(0) \equiv \Sigma_{k}(0)
$$

are familiar classes of starlike and convex functions in $U^{*}$, respectively.

Definition 1.3 Let a function $f \in \Sigma$ be analytic in $\mathbb{U}^{*}$. Then $f$ is in the class $\Sigma^{\star} \mathcal{U}(\alpha, \delta, b)$ if, and only if, $f$ satisfies

$$
\Re\left\{1-\frac{1}{b}\left(\frac{z f^{\prime}(z)}{f(z)}+1\right)\right\}>\alpha\left|\frac{1}{b}\left(\frac{z f^{\prime}(z)}{f(z)}+1\right)\right|+\delta,
$$

where $\alpha \geq 0, \delta \in[-1,1), \alpha+\delta \geq 0, b \in \mathbb{C} \backslash\{0\}$.

Definition 1.4 Let a function $f \in \Sigma$ be analytic in $U^{*}$. Then $f$ is in the class $\Sigma K \mathcal{U}(\alpha, \delta, b)$ if, and only if, $f$ satisfies

$$
\Re\left\{1-\frac{1}{b}\left(\frac{z f^{\prime \prime}(z)}{f^{\prime}(z)}+2\right)\right\}>\alpha\left|\frac{1}{b}\left(\frac{z f^{\prime \prime}(z)}{f^{\prime}(z)}+2\right)\right|+\delta,
$$

where $\alpha \geq 0, \delta \in[-1,1), \alpha+\delta \geq 0, b \in \mathbb{C} \backslash\{0\}$.

We note that $f \in \Sigma K \mathcal{U}(\alpha, \delta, b)$ if, and only if, $-z f^{\prime} \in \Sigma^{\star} \mathcal{U}(\alpha, \delta, b)$.

For $\alpha=0$, we have

$$
\Sigma^{\star} \mathcal{U}(0, \delta, b) \equiv \Sigma_{b}^{\star}(\delta), \quad \Sigma K \mathcal{U}(0, \delta, b) \equiv \Sigma K_{b}(\delta) .
$$

Definition 1.5 Let a function $f \in \Sigma$ be analytic in $U^{*}$. Then $f$ is in the class $\Sigma^{\star} \mathcal{U H}(\alpha, b)$ if, and only if, $f$ satisfies

$$
\left|1-\frac{1}{b}\left(\frac{z f^{\prime}(z)}{f(z)}+1\right)-2 \alpha(\sqrt{2}-1)\right|<\Re\left\{\sqrt{2}\left(1-\frac{1}{b}\left(\frac{z f^{\prime}(z)}{f(z)}+1\right)\right)\right\}+2 \alpha(\sqrt{2}-1),
$$

where $\alpha>0, b \in \mathbb{C} \backslash\{0\}$. 
Definition 1.6 Let a function $f \in \Sigma$ be analytic in $\mathbb{U}^{*}$. Then $f$ is in the class $\Sigma K \mathcal{U H}(\alpha, b)$ if, and only if, $f$ satisfies

$$
\left|1-\frac{1}{b}\left(\frac{z f^{\prime \prime}(z)}{f^{\prime}(z)}+2\right)-2 \alpha(\sqrt{2}-1)\right|<\Re\left\{\sqrt{2}\left(1-\frac{1}{b}\left(\frac{z f^{\prime \prime}(z)}{f^{\prime}(z)}+2\right)\right)\right\}+2 \alpha(\sqrt{2}-1) .
$$

where $\alpha>0, b \in \mathbb{C} \backslash\{0\}$.

We note that $f \in \Sigma \operatorname{KUH}(\alpha, b)$ if, and only if, $-z f^{\prime} \in \Sigma^{\star} \mathcal{U H}(\alpha, b)$.

Let us also introduce the following families of new subclasses $\Sigma \mathcal{F}_{1}(\delta, b), \Sigma \mathcal{F}_{2}(\alpha, \delta, b)$, and $\Sigma \mathcal{F}_{3}(\alpha, b)$ as follows.

Definition 1.7 Let a function $f \in \Sigma$ be analytic in $U^{*}$. Then $f$ is in the class $\Sigma \mathcal{F}_{1}(\delta, b)$, if, and only if, $f$ satisfies

$$
\Re\left\{1-\frac{1}{b}\left(\frac{z\left(z f^{\prime \prime}(z)+3 f^{\prime}(z)\right)}{z f^{\prime}(z)+2 f(z)}+1\right)\right\}>\delta .
$$

where $b \in \mathbb{C} \backslash\{0\}, 0 \leq \delta<1$.

We note that $f \in \Sigma \mathcal{F}_{1}(\delta, b)$ if, and only if, $z f^{\prime}(z)+2 f(z) \in \Sigma_{b}^{\star}(\delta)$.

Definition 1.8 Let a function $f \in \Sigma$ be analytic in $U^{*}$. Then $f$ is in the class $\Sigma \mathcal{F}_{2}(\alpha, \delta, b)$ if, and only if, $f$ satisfies

$$
\Re\left\{1-\frac{1}{b}\left(\frac{z\left(z f^{\prime \prime}(z)+3 f^{\prime}(z)\right)}{z f^{\prime}(z)+2 f(z)}+1\right)\right\}>\alpha\left|\frac{1}{b}\left(\frac{z\left(z f^{\prime \prime}(z)+3 f^{\prime}(z)\right)}{z f^{\prime}(z)+2 f(z)}+1\right)\right|+\delta,
$$

where $\alpha \geq 0, \delta \in[-1,1), \alpha+\delta \geq 0, b \in \mathbb{C} \backslash\{0\}$.

We note that $f \in \Sigma \mathcal{F}_{2}(\alpha, \delta, b)$ if, and only if, $z f^{\prime}(z)+2 f(z) \in \Sigma^{\star} \mathcal{U}(\alpha, \delta, b)$.

Definition 1.9 Let a function $f \in \Sigma$ be analytic in $U^{*}$. Then $f$ is in the class $\Sigma \mathcal{F}_{3}(\alpha, b)$ if, and only if, $f$ satisfies

$$
\left|1-\frac{1}{b}\left(\frac{z\left(z f^{\prime \prime}(z)+3 f^{\prime}(z)\right)}{z f^{\prime}(z)+2 f(z)}+1\right)-2 \alpha(\sqrt{2}-1)\right|<\Re\left\{\begin{array}{l}
\left.<\sqrt{2}\left(1-\frac{1}{b}\left(\frac{z\left(z f^{\prime \prime}(z)+3 f^{\prime}(z)\right)}{z f^{\prime}(z)+2 f(z)}+1\right)\right)\right\} \\
+2 \alpha(\sqrt{2}-1),
\end{array}\right.
$$

where $\alpha>0, b \in \mathbb{C} \backslash\{0\}$.

We note that $f \in \Sigma \mathcal{F}_{3}(\alpha, b)$ if, and only if, $z f^{\prime}(z)+2 f(z) \in \Sigma^{\star} \mathcal{U H}(\alpha, b)$.

Recently, many authors introduced and studied various integral operators of analytic and univalent functions in the open unit disk U[11-21].

Most recently, Mohammed and Darus [22,23] introduced the following two general integral operators of meromorphic functions $\Sigma$ :

$$
\mathcal{H}_{n}(z)=\frac{1}{z^{2}} \int_{0}^{z}\left(u f_{1}(u)\right)^{\gamma_{1}} \ldots\left(u f_{n}(u)\right)^{\gamma_{n}} d u,
$$

and

$$
\mathcal{H}_{\gamma_{1}, \ldots, \gamma_{n}}(z)=\frac{1}{z^{2}} \int_{0}^{z}\left(-u^{2} f_{1}^{\prime}(u)\right)^{\gamma_{1}} \ldots\left(-u^{2} f_{n}^{\prime}(u)\right)^{\gamma_{n}} d u .
$$

Goyal and Prajapat [24] obtained the following results for $f \in \Sigma$ to be in the class $\Sigma^{*}$ $(\delta), 0 \leq \delta<1$. 
Corollary 1.1 If $f \in \Sigma$ satisfies the following inequality

$$
\left|\frac{z f^{\prime \prime}(z)}{f^{\prime}(z)}-\frac{2 z f^{\prime}(z)}{f(z)}\right|<\frac{(1-\delta)(3-\delta)}{2-\delta}, \quad 0 \leq \delta<1,
$$

then $f \in \Sigma^{*}(\delta)$.

Corollary 1.2 If $f \in \Sigma$ satisfies the following inequality

$$
\left|\frac{z f^{\prime \prime}(z)}{f^{\prime}(z)}-\frac{z f^{\prime}(z)}{f(z)}+1\right|<\frac{1}{2}
$$

then $f \in \Sigma^{*}$.

Corollary 1.3 If $f \in \Sigma$ satisfies the following inequality

$$
\Re\left\{\frac{z f^{\prime}(z)}{f(z)}\left(\frac{2 z f^{\prime}(z)}{f(z)}-\frac{z f^{\prime \prime}(z)}{f^{\prime}(z)}-1\right)\right\}>-\frac{1}{2},
$$

then $f \in \Sigma^{*}$.

In this article, we derive several properties for the integral operators $\mathcal{H}_{n}(z)$ and $\mathcal{H}_{\gamma_{1}, \ldots, \gamma_{n}}(z)$ of the subclasses given by (1.5) and Definitions 1.1 to 1.6.

\section{Some properties for $\mathcal{H}_{n}(z)$}

In this section, we investigate some properties for the integral operator $\mathcal{H}_{n}(z)$ defined by (1.15) of the subclasses given by (1.5), Definitions 1.1, 1.3, and 1.5.

Theorem 2.1 For $i \in\{1, \ldots, n\}$, let $\gamma_{i}>0, f_{i} \in \Sigma$ and

$$
\left|\frac{z f_{i}^{\prime \prime}(z)}{f_{i}^{\prime}(z)}-2 \frac{z f_{i}^{\prime}(z)}{f_{i}(z)}\right|<\frac{(1-\delta)(3-\delta)}{2-\delta}, \quad(0 \leq \delta<1) .
$$

If

$$
\sum_{i=1}^{n} \gamma_{i}>\frac{2}{1-\delta}
$$

then $\mathcal{H}_{n}(z) \in \Sigma_{N}(\beta)$, where $\beta>1$.

Proof On successive differentiation of $\mathcal{H}_{n}(z)$, which is defined in (1.5), we get

$$
z^{2} \mathcal{H}_{n}^{\prime}(z)+2 z \mathcal{H}_{n}(z)=\left(z f_{1}(z)\right)^{\gamma_{1}} \ldots\left(z f_{n}(z)\right)^{\gamma_{n}},
$$

and

$$
z^{2} \mathcal{H}_{n}^{\prime \prime}(z)+4 z \mathcal{H}_{n}^{\prime}(z)+2 \mathcal{H}_{n}(z)=\sum_{i=1}^{n} \gamma_{i}\left(\frac{z f_{i}^{\prime}(z)+f_{i}(z)}{z f_{i}(z)}\right)\left[\left(z f_{1}(z)\right)^{\gamma_{1}} \ldots\left(z f_{n}(z)\right)^{\gamma_{n}}\right]
$$

Then from (2.3) and (2.4), we obtain

$$
\frac{z^{2} \mathcal{H}_{n}^{\prime \prime}(z)+4 z \mathcal{H}_{n}^{\prime}(z)+2 \mathcal{H}_{n}(z)}{z^{2} \mathcal{H}_{n}^{\prime}(z)+2 z \mathcal{H}_{n}(z)}=\sum_{i=1}^{n} \gamma_{i}\left(\frac{f_{i}^{\prime}(z)}{f_{i}(z)}+\frac{1}{z}\right) .
$$

By multiplying (2.5) with $z$ yield

$$
\frac{z^{2} \mathcal{H}_{n}^{\prime \prime}(z)+4 z \mathcal{H}_{n}^{\prime}(z)+2 \mathcal{H}_{n}(z)}{z \mathcal{H}_{n}^{\prime}(z)+2 \mathcal{H}_{n}(z)}=\sum_{i=1}^{n} \gamma_{i}\left(\frac{z f_{i}^{\prime}(z)}{f_{i}(z)}+1\right) .
$$


This is equivalent to

$$
\frac{z^{2} \mathcal{H}_{n}^{\prime \prime}(z)+3 z \mathcal{H}_{n}^{\prime}(z)}{z \mathcal{H}_{n}^{\prime}(z)+2 \mathcal{H}_{n}(z)}+1=\sum_{i=1}^{n} \gamma_{i}\left(\frac{z f_{i}^{\prime}(z)}{f_{i}(z)}+1\right)
$$

Therefore, we have

$$
\frac{-\left(\frac{z \mathcal{H}_{n}^{\prime \prime}(z)}{\mathcal{H}_{n}^{\prime}(z)}+1\right)-2}{1+\frac{2 \mathcal{H}_{n}(z)}{z \mathcal{H}_{n}^{\prime}(z)}}=-\sum_{i=1}^{n} \gamma_{i}\left(\frac{z f_{i}^{\prime}(z)}{f_{i}(z)}+1\right)+1
$$

Then, we easily get

$$
\begin{aligned}
-\left(\frac{z \mathcal{H}_{n}^{\prime \prime}(z)}{\mathcal{H}_{n}^{\prime}(z)}+1\right)= & \left(\frac{2 \mathcal{H}_{n}(z)}{z \mathcal{H}_{n}^{\prime}(z)}\right)\left[-\sum_{i=1}^{n} \gamma_{i}\left(\frac{z f_{i}^{\prime}(z)}{f_{i}(z)}+1\right)+1\right] \\
& +\sum_{i=1}^{n} \gamma_{i}\left(-\frac{z f_{i}^{\prime}(z)}{f_{i}(z)}\right)+3-\sum_{i=1}^{n} \gamma_{i} .
\end{aligned}
$$

Taking real parts of both sides of (2.9), we obtain

$$
\begin{aligned}
-\Re\left(\frac{z \mathcal{H}_{n}^{\prime \prime}(z)}{\mathcal{H}_{n}^{\prime}(z)}+1\right)= & \Re\left\{\left(\frac{2 \mathcal{H}_{n}(z)}{z \mathcal{H}_{n}^{\prime}(z)}\right)\left[-\sum_{i=1}^{n} \gamma_{i}\left(\frac{z f_{i}^{\prime}(z)}{f_{i}(z)}+1\right)+1\right]\right\} \\
& +\sum_{i=1}^{n} \gamma_{i} \Re\left(-\frac{z f_{i}^{\prime}(z)}{f_{i}(z)}\right)+3-\sum_{i=1}^{n} \gamma_{i} \\
\leq & \left|\left(\frac{2 \mathcal{H}_{n}(z)}{z \mathcal{H}_{n}^{\prime}(z)}\right)\left[-\sum_{i=1}^{n} \gamma_{i}\left(\frac{z f_{i}^{\prime}(z)}{f_{i}(z)}+1\right)+1\right]\right| \\
& +\sum_{i=1}^{n} \gamma_{i} \Re\left(-\frac{z f_{i}^{\prime}(z)}{f_{i}(z)}\right)+3-\sum_{i=1}^{n} \gamma_{i} .
\end{aligned}
$$

Let

$$
\begin{aligned}
\beta= & \left|\left(\frac{2 \mathcal{H}_{n}(z)}{z \mathcal{H}_{n}^{\prime}(z)}\right)\left[-\sum_{i=1}^{n} \gamma_{i}\left(\frac{z f_{i}^{\prime}(z)}{f_{i}(z)}+1\right)+1\right]\right| \\
& +\sum_{i=1}^{n} \gamma_{i} \Re\left(-\frac{z f_{i}^{\prime}(z)}{f_{i}(z)}\right)+3-\sum_{i=1}^{n} \gamma_{i} .
\end{aligned}
$$

Since $\left|\left(\frac{2 \mathcal{H}_{n}(z)}{z \mathcal{H}_{n}^{\prime}(z)}\right)\left[-\sum_{i=1}^{n} \gamma_{i}\left(\frac{z f_{i}^{\prime}(z)}{f_{i}(z)}+1\right)+1\right]\right|>0$, applying Corollary 1.1, we have

$$
\beta>\delta \sum_{i=1}^{n} \gamma_{i}+3-\sum_{i=1}^{n} \gamma_{i}=3-(1-\delta) \sum_{i=1}^{n} \gamma_{i}
$$

Then, by the hypothesis (2.2), we have $\beta>1$. Therefore, $\mathcal{H}_{n}(z) \in \Sigma_{N}(\beta)$, where $\beta>$ 1. This completes the proof.

Letting $\delta=0$ in Theorem 2.1, we have 
Corollary 2.2 For $i \in\{1, \ldots, n\}$, let $\gamma_{i}>0, f_{i} \in \Sigma$ and

$$
\left|\frac{z f_{i}^{\prime \prime}(z)}{f_{i}^{\prime}(z)}-2 \frac{z f_{i}^{\prime}(z)}{f_{i}(z)}\right|<\frac{3}{2} \text {. }
$$

If

$$
\sum_{i=1}^{n} \gamma_{i}>2
$$

then $\mathcal{H}_{n}(z) \in \Sigma_{N}(\beta)$, where $\beta>1$.

Making use of (2.11), Corollary 1.2 and Corollary 1.3, one can prove the following results.

Theorem 2.3 For $i \in\{1, \ldots, n\}$, let $\gamma_{i}>0, f_{i} \in \Sigma$ and

$$
\left|\frac{z f_{i}^{\prime \prime}(z)}{f_{i}^{\prime}(z)}-\frac{z f_{i}^{\prime}(z)}{f_{i}(z)}+1\right|<\frac{1}{2}
$$

If

$$
\sum_{i=1}^{n} \gamma_{i}>2
$$

then $\mathcal{H}_{n}(z) \in \Sigma_{N}(\beta)$, where $\beta>1$.

Theorem 2.4 For $i \in\{1, \ldots, n\}$, let $\gamma_{i}>0, f_{i} \in \Sigma$ and

$$
\Re\left\{\frac{z f_{i}^{\prime}(z)}{f_{i}(z)}\left(\frac{2 z f_{i}^{\prime}(z)}{f_{i}(z)}-\frac{z f_{i}^{\prime \prime}(z)}{f_{i}^{\prime}(z)}-1\right)\right\}>-\frac{1}{2} .
$$

If

$$
\sum_{i=1}^{n} \gamma_{i}>2
$$

then $\mathcal{H}_{n}(z) \in \Sigma_{N}(\beta)$, where $\beta>1$.

Theorem 2.5 For $i \in\{1, \ldots, n\}$, let $\gamma_{i}>0$ and $f_{i} \in \Sigma_{b}^{\star}\left(\delta_{i}\right)(0 \leq \delta<1$ and $b \in \mathbb{C} \backslash\{0\})$.

If

$$
0<\sum_{i=1}^{n} \gamma_{i}\left(1-\delta_{i}\right) \leq 1
$$

then $\mathcal{H}_{n}(z)$ is in the class $\Sigma \mathcal{F}_{1}(\mu, b), \quad \mu=1-\sum_{i=1}^{n} \gamma_{i}\left(1-\delta_{i}\right)$.

Proof From (2.7), we have

$$
\frac{z\left(z \mathcal{H}_{n}^{\prime \prime}(z)+3 \mathcal{H}_{n}^{\prime}(z)\right)}{z \mathcal{H}_{n}^{\prime}(z)+2 \mathcal{H}_{n}(z)}+1=\sum_{i=1}^{n} \gamma_{i}\left(\frac{z f_{i}^{\prime}(z)}{f_{i}(z)}+1\right) .
$$

Equivalently, (2.20) can be written as

$$
1-\frac{1}{b}\left\{\frac{z\left(z \mathcal{H}_{n}^{\prime \prime}(z)+3 \mathcal{H}_{n}^{\prime}(z)\right)}{z \mathcal{H}_{n}^{\prime}(z)+2 \mathcal{H}_{n}(z)}+1\right\}=\sum_{i=1}^{n} \gamma_{i}\left\{1-\frac{1}{b}\left(\frac{z f_{i}^{\prime}(z)}{f_{i}(z)}+1\right)\right\}+1-\sum_{i=1}^{n} \gamma_{i} .
$$


Taking the real part of both terms of the last expression, we have

$$
\Re\left\{1-\frac{1}{b}\left(\frac{z\left(z \mathcal{H}_{n}^{\prime \prime}(z)+3 \mathcal{H}_{n}^{\prime}(z)\right)}{z \mathcal{H}_{n}^{\prime}(z)+2 \mathcal{H}_{n}(z)}+1\right)\right\}=\sum_{i=1}^{n} \gamma_{i} \Re\left\{1-\frac{1}{b}\left(\frac{z f_{i}^{\prime}(z)}{f_{i}(z)}+1\right)\right\}+1-\sum_{i=1}^{n} \gamma_{i} .
$$

Since $f_{i} \in \Sigma_{b}^{\star}\left(\delta_{i}\right)$, hence

$$
\Re\left\{1-\frac{1}{b}\left(\frac{z\left(z \mathcal{H}_{n}^{\prime \prime}(z)+3 \mathcal{H}_{n}^{\prime}(z)\right)}{z \mathcal{H}_{n}^{\prime}(z)+2 \mathcal{H}_{n}(z)}+1\right)\right\}>\sum_{i=1}^{n} \gamma_{i} \delta_{i}+1-\sum_{i=1}^{n} \gamma_{i} .
$$

so that

$$
\Re\left\{1-\frac{1}{b}\left(\frac{z\left(z \mathcal{H}_{n}^{\prime \prime}(z)+3 \mathcal{H}_{n}^{\prime}(z)\right)}{z \mathcal{H}_{n}^{\prime}(z)+2 \mathcal{H}_{n}(z)}+1\right)\right\}>1-\sum_{i=1}^{n} \gamma_{i}\left(1-\delta_{i}\right) .
$$

Then $\mathcal{H}_{n}(z) \in \Sigma \mathcal{F}_{1}(\mu, b), \quad \mu=1-\sum_{i=1}^{n} \gamma_{i}\left(1-\delta_{i}\right)$.

Now, adopting the techniques used by Breaz et al. [11] and Bulut [21], we prove the following two theorems.

Theorem 2.6 For $i \in\{1, \ldots, n\}$, let $\gamma_{i}>0$ and $f_{i} \in \Sigma^{\star} \mathcal{U}(\alpha, \delta, b)(\alpha \geq 0, \delta \in[-1,1), \alpha+$ $\delta \geq 0$ and $b \in \mathbb{C} \backslash\{0\})$. If

$$
\sum_{i=1}^{n} \gamma_{i} \leq 1
$$

then $\mathcal{H}_{n}(z)$ is in the class $\Sigma \mathcal{F}_{2}(\alpha, \delta, b)$.

Proof Since $f_{i} \in \Sigma^{\star} \mathcal{U}(\alpha, \delta, b)$, it follows from Definition 1.3 that

$$
\Re\left\{1-\frac{1}{b}\left(\frac{z f_{i}^{\prime}(z)}{f_{i}(z)}+1\right)\right\}>\alpha\left|\frac{1}{b}\left(\frac{z f_{i}^{\prime}(z)}{f_{i}(z)}+1\right)\right|+\delta .
$$

Considering Definition 1.8 and with the help of (2.21), we obtain

$$
\begin{aligned}
& \Re\left\{1-\frac{1}{b}\left(\frac{z\left(z \mathcal{H}_{n}^{\prime \prime}(z)+3 \mathcal{H}_{n}^{\prime}(z)\right)}{z \mathcal{H}_{n}^{\prime}(z)+2 \mathcal{H}_{n}(z)}+1\right)\right\}-\alpha\left|\frac{1}{b}\left(\frac{z\left(z \mathcal{H}_{n}^{\prime \prime}(z)+3 \mathcal{H}_{n}^{\prime}(z)\right)}{z \mathcal{H}_{n}^{\prime}(z)+2 \mathcal{H}_{n}(z)}+1\right)\right|-\delta \\
& \quad=1-\sum_{i=1}^{n} \gamma_{i}+\sum_{i=1}^{n} \gamma_{i} \Re\left\{1-\frac{1}{b}\left(\frac{z f_{i}^{\prime}(z)}{f_{i}(z)}+1\right)\right\}-\alpha\left|\sum_{i=1}^{n} \gamma_{i} \frac{1}{b}\left(\frac{z f_{i}^{\prime}(z)}{f_{i}(z)}+1\right)\right|-\delta \\
& \quad \geq 1-\sum_{i=1}^{n} \gamma_{i}+\sum_{i=1}^{n} \gamma_{i} \Re\left\{1-\frac{1}{b}\left(\frac{z f_{i}^{\prime}(z)}{f_{i}(z)}+1\right)\right\}-\alpha \sum_{i=1}^{n} \gamma_{i}\left|\frac{1}{b}\left(\frac{z f_{i}^{\prime}(z)}{f_{i}(z)}+1\right)\right|-\delta \\
& \quad>1-\sum_{i=1}^{n} \gamma_{i}+\sum_{i=1}^{n} \gamma_{i}\left\{\alpha\left|\frac{1}{b}\left(\frac{z f_{i}^{\prime}(z)}{f_{\prime}(z)}+1\right)\right|+\delta\right\}-\alpha \sum_{i=1}^{n} \gamma_{i}\left|\frac{1}{b}\left(\frac{z f_{i}^{\prime}(z)}{f_{i}(z)}+1\right)\right|-\delta \\
& =(1-\delta)\left(1-\sum_{i=1}^{n} \gamma_{i}\right) \geq 0 .
\end{aligned}
$$

This completes the proof. $\quad \square$

Theorem 2.7 For $i \in\{1, \ldots, n\}$, let $\gamma_{i}>0$ and $f_{i} \in \Sigma^{\star} \mathcal{U H}(\alpha, b)(\alpha>0$ and $b \in \mathbb{C} \backslash\{0\})$. If 


$$
\sum_{i=1}^{n} \gamma_{i} \leq 1,
$$

then $\mathcal{H}_{n}(z)$ is in the class $\Sigma \mathcal{F}_{3}(\alpha, b)$.

Proof Since $f_{i} \in \Sigma^{\star} \mathcal{U H}(\alpha, b)$, it follows from Definition 1.5 that

$$
\Re\left\{\sqrt{2}\left(1-\frac{1}{b}\left(\frac{z f_{i}^{\prime}(z)}{f_{i}(z)}+1\right)\right)\right\}+2 \alpha(\sqrt{2}-1)-\left|1-\frac{1}{b}\left(\frac{z f_{i}^{\prime}(z)}{f_{i}(z)}+1\right)-2 \alpha(\sqrt{2}-1)\right|>0 .
$$

Considering this inequality and (2.21), we obtain

$$
\begin{aligned}
& \Re\left\{\sqrt{2}\left(1-\frac{1}{b}\left(\frac{z\left(z \mathcal{H}_{n}^{\prime \prime}(z)+3 \mathcal{H}_{n}^{\prime}(z)\right)}{z \mathcal{H}_{n}^{\prime}(z)+2 \mathcal{H}_{n}(z)}+1\right)\right)\right\}+2 \alpha(\sqrt{2}-1)-\mid 1-\frac{1}{b}\left(\frac{z\left(z \mathcal{H}_{n}^{\prime \prime}(z)+3 \mathcal{H}_{n}^{\prime}(z)\right)}{z \mathcal{H}_{n}^{\prime}(z)+2 \mathcal{H}_{n}(z)}+1\right) \\
& -2 \alpha(\sqrt{2}-1) \\
& =\Re\left\{\sqrt{2}\left[1-\sum_{i=1}^{n} \gamma_{i} \frac{1}{b}\left(\frac{z f_{i}^{\prime}(z)}{f_{i}(z)}+1\right)\right]\right\}+2 \alpha(\sqrt{2}-1)-\mid 1-\sum_{i=1}^{n} \gamma_{i} \frac{1}{b}\left(\frac{z f_{i}^{\prime}(z)}{f_{i}(z)}+1\right) \\
& -2 \alpha(\sqrt{2}-1) \\
& =\sqrt{2}-\sqrt{2} \sum_{i=1}^{n} \gamma_{i} \Re \frac{1}{b}\left(\frac{z f_{i}^{\prime}(z)}{f_{i}(z)}+1\right)+2 \alpha(\sqrt{2}-1)-\mid 1-\sum_{i=1}^{n} \gamma_{i} \frac{1}{b}\left(\frac{z f_{i}^{\prime}(z)}{f_{i}(z)}+1\right) \\
& -2 \alpha(\sqrt{2}-1) \\
& =\sqrt{2}+\sqrt{2} \sum_{i=1}^{n} \gamma_{i} \Re\left\{1-\frac{1}{b}\left(\frac{z f_{i}^{\prime}(z)}{f_{i}(z)}+1\right)\right\}-\sqrt{2} \sum_{i=1}^{n} \gamma_{i}+2 \alpha(\sqrt{2}-1) \\
& -\mid 1+\sum_{i=1}^{n} \gamma_{i}\left[1-\frac{1}{b}\left(\frac{z f_{i}^{\prime}(z)}{f_{i}(z)}+1\right)-2 \alpha(\sqrt{2}-1)\right]-\sum_{i=1}^{n} \gamma_{i}+2 \alpha(\sqrt{2}-1) \sum_{i=1}^{n} \gamma_{i} \\
& -2 \alpha(\sqrt{2}-1) \\
& =\sqrt{2}\left(1-\sum_{i=1}^{n} \gamma_{i}\right)+2 \alpha(\sqrt{2}-1)+\sqrt{2} \sum_{i=1}^{n} \gamma_{i} \Re\left\{1-\frac{1}{b}\left(\frac{z f_{i}^{\prime}(z)}{f_{i}(z)}+1\right)\right\} \\
& -\left|[1-2 \alpha(\sqrt{2}-1)]\left(1-\sum_{i=1}^{n} \gamma_{i}\right)+\sum_{i=1}^{n} \gamma_{i}\left[1-\frac{1}{b}\left(\frac{z f_{i}^{\prime}(z)}{f_{i}(z)}+1\right)-2 \alpha(\sqrt{2}-1)\right]\right| \\
& \geq \sqrt{2}\left(1-\sum_{i=1}^{n} \gamma_{i}\right)+2 \alpha(\sqrt{2}-1)+\sqrt{2} \sum_{i=1}^{n} \gamma_{i} \Re\left\{1-\frac{1}{b}\left(\frac{z f_{i}^{\prime}(z)}{f_{i}(z)}+1\right)\right\} \\
& -\sum_{i=1}^{n} \gamma_{i}\left|1-\frac{1}{b}\left(\frac{z f_{i}^{\prime}(z)}{f_{i}(z)}+1\right)-2 \alpha(\sqrt{2}-1)\right|-|1-2 \alpha(\sqrt{2}-1)|\left(1-\sum_{i=1}^{n} \gamma_{i}\right) \\
& =\sum_{i=1}^{n} \gamma_{i}\left\{\Re \sqrt{2}\left[1-\frac{1}{b}\left(\frac{z f_{i}^{\prime}(z)}{f_{i}(z)}+1\right)\right]+2 \alpha(\sqrt{2}-1)-\left|1-\frac{1}{b}\left(\frac{z f_{i}^{\prime}(z)}{f_{i}(z)}+1\right)-2 \alpha(\sqrt{2}-1)\right|\right\} \\
& +\sqrt{2}\left(1-\sum_{i=1}^{n} \gamma_{i}\right)+2 \alpha(\sqrt{2}-1)-2 \alpha(\sqrt{2}-1) \sum_{i=1}^{n} \gamma_{i}-|1-2 \alpha(\sqrt{2}-1)|\left(1-\sum_{i=1}^{n} \gamma_{i}\right) \\
& >[\sqrt{2}+2 \alpha(\sqrt{2}-1)-|1-2 \alpha(\sqrt{2}-1)|]\left(1-\sum_{i=1}^{n} \gamma_{i}\right) \\
& >\left(1-\sum_{i=1}^{n} \gamma_{i}\right) \min \{(\sqrt{2}-1)(1+4 \alpha), \sqrt{2}+1\} \geq 0 .
\end{aligned}
$$

This completes the proof. $\square$

\section{Some properties for $\mathcal{H}_{\gamma_{1}, \ldots, \gamma_{n}}(z)$}

In this section, we investigate some properties for the integral operator $\mathcal{H}_{\gamma_{1}, \ldots, \gamma_{n}}(z)$ defined by (1.16) of subclasses given by (1.5), Definitions 1.2, 1.4, and 1.6.

Theorem 3.1 For $i \in\{1, \ldots, n\}$, let $\gamma_{i}>0, f_{i} \in \Sigma$ and

$$
\sum_{i=1}^{n} \gamma_{i}>\frac{2}{1-\delta}, \quad(0 \leq \delta<1) \text {. }
$$

If $f_{i} \in \Sigma_{k}(\delta)$, then $\mathcal{H}_{\gamma_{1}, \ldots, \gamma_{n}}(z) \in \Sigma_{N}(\beta), \beta>1$. 
Proof On successive differentiation of $\mathcal{H}_{\gamma_{1}, \ldots, \gamma_{n}}(z)$, which is defined in (1.16), we have

$$
2 z \mathcal{H}_{\gamma_{1}, \ldots, \gamma_{n}}(z)+z^{2} \mathcal{H}_{\gamma_{1}, \ldots, \gamma_{n}}^{\prime}(z)=\left(-z^{2} f_{1}^{\prime}(z)\right)^{\gamma_{1}} \ldots\left(-z^{2} f_{n}^{\prime}(z)\right)^{\gamma_{n}},
$$

and

$$
\begin{aligned}
& z^{2} \mathcal{H}_{\gamma_{1}, \ldots, \gamma_{n}}^{\prime \prime}(z)+4 z \mathcal{H}_{\gamma_{1}, \ldots, \gamma_{n}}^{\prime}(z)+2 \mathcal{H}_{\gamma_{1}, \ldots, \gamma_{n}}(z) \\
& =\sum_{i=1}^{n} \gamma_{i}\left(\frac{f_{i}^{\prime \prime}(z)}{f_{i}^{\prime}(z)}+\frac{2}{z}\right)\left[\left(-z^{2} f_{1}^{\prime}(z)\right)^{\gamma_{1}} \ldots\left(-z^{2} f_{n}^{\prime}(z)\right)^{\gamma_{n}}\right] .
\end{aligned}
$$

Then from (3.2) and (3.3), we obtain

$$
\frac{z^{2} \mathcal{H}_{\gamma_{1}, \ldots, \gamma_{n}}^{\prime \prime}(z)+4 z \mathcal{H}_{\gamma_{1}, \ldots, \gamma_{n}}^{\prime}(z)+2 \mathcal{H}_{\gamma_{1}, \ldots, \gamma_{n}}(z)}{z^{2} \mathcal{H}_{\gamma_{1}, \ldots, \gamma_{n}}^{\prime}(z)+2 z \mathcal{H}_{\gamma_{1}, \ldots, \gamma_{n}}(z)}=\sum_{i=1}^{n} \gamma_{i}\left(\frac{f_{i}^{\prime \prime}(z)}{f_{i}^{\prime}(z)}+\frac{2}{z}\right) .
$$

By multiplying (3.4) with $z$ yields,

$$
\frac{z^{2} \mathcal{H}_{\gamma_{1}, \ldots, \gamma_{n}}^{\prime \prime}(z)+4 z \mathcal{H}_{\gamma_{1}, \ldots, \gamma_{n}}^{\prime}(z)+2 \mathcal{H}_{\gamma_{1}, \ldots, \gamma_{n}}(z)}{z \mathcal{H}_{\gamma_{1}, \ldots, \gamma_{n}}^{\prime}(z)+2 \mathcal{H}_{\gamma_{1}, \ldots, \gamma_{n}}(z)}=\sum_{i=1}^{n} \gamma_{i}\left(\frac{z f_{i}^{\prime \prime}(z)}{f_{i}^{\prime}(z)}+2\right) \text {. }
$$

that is equivalent to

$$
\frac{z^{2} \mathcal{H}_{\gamma_{1}, \ldots, \gamma_{n}}^{\prime \prime}(z)+3 z \mathcal{H}_{\gamma_{1}, \ldots, \gamma_{n}}^{\prime}(z)}{z \mathcal{H}_{\gamma_{1}, \ldots, \gamma_{n}}^{\prime}(z)+2 \mathcal{H}_{\gamma_{1}, \ldots, \gamma_{n}}(z)}+1=\sum_{i=1}^{n} \gamma_{i}\left(\frac{z f_{i}^{\prime \prime}(z)}{f_{i}^{\prime}(z)}+2\right)
$$

Therefore, we have

$$
\frac{-\left(\frac{z \mathcal{H}_{\gamma_{1}, \ldots, \gamma_{n}}^{\prime \prime}(z)}{\mathcal{H}_{\gamma_{1}, \ldots, \gamma_{n}}^{\prime}(z)}+1\right)-2}{1+\frac{2 \mathcal{H}_{\gamma_{1}, \ldots, \gamma_{n}}(z)}{z \mathcal{H}_{\gamma_{1}, \ldots, \gamma_{n}}^{\prime}(z)}}=-\sum_{i=1}^{n} \gamma_{i}\left(\frac{z f_{i}^{\prime \prime}(z)}{f_{i}^{\prime}(z)}+2\right)+1 .
$$

so that

$$
\begin{aligned}
-\left(\frac{z \mathcal{H}_{\gamma_{1}, \ldots, \gamma_{n}}^{\prime \prime}(z)}{\mathcal{H}_{\gamma_{1}, \ldots, \gamma_{n}}^{\prime}(z)}+1\right)= & \left(\frac{2 \mathcal{H}_{\gamma_{1}, \ldots, \gamma_{n}}(z)}{z \mathcal{H}_{\gamma_{1}, \ldots, \gamma_{n}}^{\prime}(z)}\right)\left[-\sum_{i=1}^{n} \gamma_{i}\left(\frac{z f_{i}^{\prime \prime}(z)}{f_{i}^{\prime}(z)}+2\right)+1\right] \\
& +\sum_{i=1}^{n} \gamma_{i}\left\{-\left(\frac{z f_{i}^{\prime \prime}(z)}{f_{i}^{\prime}(z)}+1\right)\right\}+3-\sum_{i=1}^{n} \gamma_{i} .
\end{aligned}
$$

Taking the real parts of both terms of the last expression, we obtain

$$
\begin{aligned}
-\Re\left(\frac{z \mathcal{H}_{\gamma_{1}, \ldots, \gamma_{n}}^{\prime \prime}(z)}{\mathcal{H}_{\gamma_{1}, \ldots, \gamma_{n}}^{\prime}(z)}+1\right)= & \Re\left\{\frac{2 \mathcal{H}_{\gamma_{1}, \ldots, \gamma_{n}}(z)}{z \mathcal{H}_{\gamma_{1}, \ldots, \gamma_{n}}^{\prime}(z)}\left[-\sum_{i=1}^{n} \gamma_{i}\left(\frac{z f_{i}^{\prime \prime}(z)}{f_{i}^{\prime}(z)}+2\right)+1\right]\right\} \\
& +\sum_{i=1}^{n} \gamma_{i} \Re\left\{-\left(\frac{z f_{i}^{\prime \prime}(z)}{f_{i}^{\prime}(z)}+1\right)\right\}+3-\sum_{i=1}^{n} \gamma_{i} \\
\leq & \frac{2 \mathcal{H}_{\gamma_{1}, \ldots, \gamma_{n}}(z)}{z \mathcal{H}_{\gamma_{1}, \ldots, \gamma_{n}}^{\prime}(z)}\left[-\sum_{i=1}^{n} \gamma_{i}\left(\frac{z f_{i}^{\prime \prime}(z)}{f_{i}^{\prime}(z)}+2\right)+1\right] \mid \\
& +\sum_{i=1}^{n} \gamma_{i} \Re\left\{-\left(\frac{z f_{i}^{\prime \prime}(z)}{f_{i}^{\prime}(z)}+1\right)\right\}+3-\sum_{i=1}^{n} \gamma_{i} .
\end{aligned}
$$


Let

$$
\beta=\left|\frac{2 \mathcal{H}_{\gamma_{1}, \ldots, \gamma_{n}}(z)}{z \mathcal{H}_{\gamma_{1}, \ldots, \gamma_{n}}^{\prime}(z)}\left[-\sum_{i=1}^{n} \gamma_{i}\left(\frac{z f_{i}^{\prime \prime}(z)}{f_{i}^{\prime}(z)}+2\right)+1\right]\right|+\sum_{i=1}^{n} \gamma_{i} \Re\left\{-\left(\frac{z f_{i}^{\prime \prime}(z)}{f_{i}^{\prime}(z)}+1\right)\right\}+3-\sum_{i=1}^{n} \gamma_{i} .
$$

Since $\left|\frac{2 \mathcal{H}_{\gamma_{1}, \ldots, \gamma_{n}}(z)}{z \mathcal{H}_{\gamma_{1}, \ldots, \gamma_{n}}^{\prime}(z)}\left[-\sum_{i=1}^{n} \gamma_{i}\left(\frac{z f_{i}^{\prime \prime}(z)}{f_{i}^{\prime}(z)}+2\right)+1\right]\right|>0, f_{i} \in \Sigma_{k}(\delta)$, we get

$$
\beta>3-(1-\delta) \sum_{i=1}^{n} \gamma_{i}
$$

which, in light of the hypothesis (3.1), yields $\beta>1$.

Therefore, $\mathcal{H}_{\gamma_{1}, \ldots, \gamma_{n}}(z) \in \Sigma_{N}(\beta), \beta>1$. This completes the proof. $\quad \square$

Theorem 3.2 For $i \in\{1, \ldots, n\}$, let $\gamma_{i}>0, f_{i} \in \Sigma$ and

$$
1<\sum_{i=1}^{n} \gamma_{i}<2
$$

If $\mathcal{H}_{\gamma_{1}, \ldots, \gamma_{n}}(z) \in \Sigma^{\star}(\delta)$, then $\mathcal{H}_{\gamma_{1}, \ldots, \gamma_{n}}(z) \in \Sigma_{N}(\beta), \beta>1$.

Proof It follows from (3.7) that

$$
\begin{aligned}
-\left(\frac{z \mathcal{H}_{\gamma_{1}, \ldots, \gamma_{n}}^{\prime \prime}(z)}{\mathcal{H}_{\gamma_{1}, \ldots, \gamma_{n}}^{\prime}(z)}+1\right)= & \left(1+\frac{2 \mathcal{H}_{\gamma_{1}, \ldots, \gamma_{n}}(z)}{z \mathcal{H}_{\gamma_{1}, \ldots, \gamma_{n}}^{\prime}(z)}\right)\left[-\sum_{i=1}^{n} \gamma_{i}\left(\frac{z f_{i}^{\prime \prime}(z)}{f_{i}^{\prime}(z)}+1\right)\right] \\
& +2\left(\sum_{i=1}^{n} \gamma_{i}-1\right)\left(-\frac{\mathcal{H}_{\gamma_{1}, \ldots, \gamma_{n}}(z)}{z \mathcal{H}_{\gamma_{1}, \ldots, \gamma_{n}}^{\prime}(z)}\right)+3-\sum_{i=1}^{n} \gamma_{i} .
\end{aligned}
$$

Taking the real parts of both terms of the last expression, we obtain

$$
\begin{aligned}
-\Re\left(\frac{z \mathcal{H}_{\gamma_{1}, \ldots, \gamma_{n}}^{\prime \prime}(z)}{\mathcal{H}_{\gamma_{1}, \ldots, \gamma_{n}}^{\prime}(z)}+1\right)= & \Re\left\{\left(1+\frac{2 \mathcal{H}_{\gamma_{1}, \ldots, \gamma_{n}}(z)}{z \mathcal{H}_{\gamma_{1}, \ldots, \gamma_{n}}^{\prime}(z)}\right)\left[-\sum_{i=1}^{n} \gamma_{i}\left(\frac{z f_{i}^{\prime \prime}(z)}{f_{i}^{\prime}(z)}+1\right)\right]\right\} \\
& +2\left(\sum_{i=1}^{n} \gamma_{i}-1\right) \Re\left(-\frac{\mathcal{H}_{\gamma_{1}, \ldots, \gamma_{n}}(z)}{z \mathcal{H}_{\gamma_{1}, \ldots, \gamma_{n}}^{\prime}(z)}\right)+3-\sum_{i=1}^{n} \gamma_{i} .
\end{aligned}
$$

Thus, we have

$$
\begin{aligned}
& -\Re\left(\frac{z \mathcal{H}_{\gamma_{1}, \ldots, \gamma_{n}}^{\prime \prime}(z)}{\mathcal{H}_{\gamma_{1}, \ldots, \gamma_{n}}^{\prime}(z)}+1\right)=\Re\left\{\left(1+\frac{2 \mathcal{H}_{\gamma_{1}, \ldots, \gamma_{n}}(z)}{z \mathcal{H}_{\gamma_{1}, \ldots, \gamma_{n}}^{\prime}(z)}\right)\left[-\sum_{i=1}^{n} \gamma_{i}\left(\frac{z f_{i}^{\prime \prime}(z)}{f_{i}^{\prime}(z)}+1\right)\right]\right\} \\
& +2\left(\sum_{i=1}^{n} \gamma_{i}-1\right) \Re\left(-\frac{1}{\frac{z \mathcal{H}_{\gamma_{1}, \ldots, \gamma_{n}}^{\prime}(z)}{\mathcal{H}_{\gamma_{1}, \ldots, \gamma_{n}}(z)}}\right)+3-\sum_{i=1}^{n} \gamma_{i} \\
& \leq\left|\left(1+\frac{2 \mathcal{H}_{\gamma_{1}, \ldots, \gamma_{n}}(z)}{z \mathcal{H}_{\gamma_{1}, \ldots, \gamma_{n}}^{\prime}(z)}\right)\left[-\sum_{i=1}^{n} \gamma_{i}\left(\frac{z f_{i}^{\prime \prime}(z)}{f_{i}^{\prime}(z)}+1\right)\right]\right| \\
& +2\left(\sum_{i=1}^{n} \gamma_{i}-1\right) \frac{\Re\left(-\frac{z \mathcal{H}_{\gamma_{1}, \ldots, \gamma_{n}}^{\prime}(z)}{\mathcal{H}_{\gamma_{1}, \ldots, \gamma_{n}}(z)}\right)}{\left|\frac{z \mathcal{H}_{\gamma_{1}, \ldots, \gamma_{n}}^{\prime}(z)}{H_{\gamma_{1}, \ldots, \gamma_{n}}(z)}\right|^{2}}+3-\sum_{i=1}^{n} \gamma_{i} .
\end{aligned}
$$


Let

$$
\begin{aligned}
\beta= & \left|\left(1+\frac{2 \mathcal{H}_{\gamma_{1}, \ldots, \gamma_{n}}(z)}{z \mathcal{H}_{\gamma_{1}, \ldots, \gamma_{n}}^{\prime}(z)}\right)\left[-\sum_{i=1}^{n} \gamma_{i}\left(\frac{z f_{i}^{\prime \prime}(z)}{f_{i}^{\prime}(z)}+1\right)\right]\right| \\
& +2\left(\sum_{i=1}^{n} \gamma_{i}-1\right) \frac{\Re\left(-\frac{z \mathcal{H}_{\gamma_{1}, \ldots, \gamma_{n}}^{\prime}(z)}{\mathcal{H}_{\gamma_{1}, \ldots, \gamma_{n}}(z)}\right)}{\left|\frac{z \mathcal{H}_{\gamma_{1}, \ldots, \gamma_{n}}^{\prime}(z)}{\mathcal{H}_{\gamma_{1}, \ldots, \gamma_{n}}(z)}\right|^{2}}+3-\sum_{i=1}^{n} \gamma_{i} .
\end{aligned}
$$

Since $\left|\left(1+\frac{2 \mathcal{H}_{\gamma_{1}, \ldots, \gamma_{n}}(z)}{z \mathcal{H}_{\gamma_{1}, \ldots, \gamma_{n}}^{\prime}(z)}\right)\left[-\sum_{i=1}^{n} \gamma_{i}\left(\frac{z f_{i}^{\prime \prime}(z)}{f_{i}^{\prime}(z)}+1\right)\right]\right|>0$ and $\mathcal{H}_{\gamma_{1}, \ldots, \gamma_{n}}(z) \in \Sigma^{\star}(\delta)$, we have

$$
\beta>2\left(\sum_{i=1}^{n} \gamma_{i}-1\right) \frac{\delta}{\left|\frac{z \mathcal{H}_{\gamma_{1}, \ldots, \gamma_{n}}^{\prime}(z)}{\mathcal{H}_{\gamma_{1}, \ldots, \gamma_{n}}(z)}\right|^{2}}+3-\sum_{i=1}^{n} \gamma_{i} .>3-\sum_{i=1}^{n} \gamma_{i}
$$

Then, by the hypothesis (3.12), we see that $\beta>1$. Therefore, $\mathcal{H}_{\gamma_{1}, \ldots, \gamma_{n}}(z) \in \Sigma_{N}(\beta), \beta$ $>1$. This completes the proof.

Now, using the method given in the proofs of Theorems 2.5, 2.6, and 2.7, one can prove the following results:

Theorem 3.3 For $i \in\{1, \ldots, n\}$, let $\gamma_{i}>0 f_{i} \in \Sigma K_{b}\left(\delta_{i}\right)(0 \leq \delta<1$ and $\left.b \in \mathbb{C} \backslash\{0\})\right)$. If

$$
0<\sum_{i=1}^{n} \gamma_{i}\left(1-\delta_{i}\right) \leq 1
$$

then $\mathcal{H}_{\gamma_{1}, \ldots, \gamma_{n}}(z)$ is in the class $\Sigma \mathcal{F}_{1}(\mu, b), \quad \mu=1-\sum_{i=1}^{n} \gamma_{i}\left(1-\delta_{i}\right)$.

Theorem 3.4 For $i \in\{1, \ldots, n\}$, let $\gamma_{i}>0$ and $f_{i} \in \Sigma K \mathcal{U}(\alpha, \delta, b)(\alpha \geq 0, \delta \in[-1,1), \alpha+$ $\delta \geq 0$ and $b \in \mathbb{C} \backslash\{0\}))$. If

$$
\sum_{i=1}^{n} \gamma_{i} \leq 1
$$

then $\mathcal{H}_{\gamma_{1}, \ldots, \gamma_{n}}(z)$ is in the class $\Sigma \mathcal{F}_{2}(\alpha, \delta, b)$.

Theorem 3.5 For $i \in\{1, \ldots, n\}$, let $\gamma_{i}>0$ and $f_{i} \in \operatorname{\Sigma KU\mathcal {H}}(\alpha, b)(\alpha \geq 0$, and $b \in \mathbb{C} \backslash\{0\}))$. If

$$
\sum_{i=1}^{n} \gamma_{i} \leq 1
$$

then $\mathcal{H}_{\gamma_{1}, \ldots, \gamma_{n}}(z)$ is in the class $\Sigma \mathcal{F}_{3}(\alpha, \delta, b)$. 
The authors declare that they have no competing interests.

Received: 21 August 2011 Accepted: 25 November 2011 Published: 25 November 2011

\section{References}

1. Wang, Z-G, Sun, Y, Zhang, Z-H: Certain classes of meromorphic multivalent functions. Comput Math Appl. 58 , 1408-1417 (2009). doi:10.1016/i.camwa.2009.07.020

2. Nehari, Z, Netanyahu, E: On the coefficients of meromorphic Schlicht functions. Proc Am Math Soc. 8, 15-23 (1957). doi:10.1090/S0002-9939-1957-0083038-0

3. Goodman, AW: Univalent Functions. Mariner, Tampa, FLI, II (1983)

4. Frasin, BA: Family of analytic functions of complex order. Acta Math Acad Paed Ny. 22(2), 179-191 (2006)

5. Ronning, F: Uniformly convex functions. Ann Polon Math. 57, 165-175 (1992)

6. Murugusundaramoorthy, G, Maghesh, N: A new subclass of uniforlmly convex functions and a corresponding subclass of starlike functions with fixed second coefficient. J Inequal Pure Appl Math. 5(4), 1-10 (2005)

7. Stankiewicz, J, Wisniowska, A: Starlike functions associated with some hyperbola. Folia Scientiarum Universitatis Tehnicae Resoviensis 147, Matematyka. 19, 117-126 (1996)

8. Acu, M: Subclasses of convex functions associated with some hyperbola. Acta Universitatis Apulensis. 12, 3-12 (2006)

9. Acu, M, Owa, S: Convex functions associated with some hyperbola. J Approx Theory Appl. 1, 37-40 (2005)

10. Nasr, MA, Aouf, MK: Starlike function of complex order. J Nat Sci Math. 25(91), 1-12 (1985)

11. Baricz, A, Frasin, BA: Univalence of integral operators involving Bessel functions. Appl Math Lett. 23, 371-376 (2010). doi:10.1016/j.aml.2009.10.013

12. Mohammed, A, Darus, M: New properties for certain integral operators. Int J Math Anal. 4(42), 2101-2109 (2010)

13. Frasin, BA: Univalence of two general integral operators. Filomat. 23, 223-229 (2009). doi:10.2298/FlL0903223F

14. Breaz, D, Breaz, N, Srivastava, HM: An extension of the univalent condition for a family of integral operators. Appl Math Lett. 22, 41-44 (2009). doi:10.1016/j.aml.2007.11.008

15. Breaz, D, Breaz, N: Two integral operators. Studia Universitatis Babes-Bolyai, Mathematica, Cluj-Napoca. 3, 13-21 (2002)

16. Breaz, D, Owa, S, Breaz, N: A new integral univalent operator. Acta Univ Apulensis Math Inform. 16, 11-16 (2008)

17. Breaz, D, Güney, HÖ, Salagean, GS: A new general integral operator. Tamsui Oxford, J Math Sci. 25(4), 407-414 (2009)

18. Darus, M, Faisal, I: A study on Beckers univalence criteria. Abs Appl Anal 2011, 13 (2011). Article, ID 759175

19. Breaz, N, Braez, D, Darus, M: Convexity properties for some general integral operators on uniformly analytic functions classes. Comput Math Appl. 60, 3105-3107 (2010). doi:10.1016/j.camwa.2010.10.012

20. Latha, S, Dileep, L: On a generalized integral operator. Int J Math Anal. 3(30), 1487-1491 (2009)

21. Bulut, S: A new general integral operator defined by Al-Oboudi differential operator. J Inequal Appl 2009, 13 (2009). Article, ID 158408

22. Mohammed, A, Darus, M: A new integral operator for meromorphic functions. Acta Universitatis Apulensis. 24, 231-238 (2010)

23. Mohammed, A, Darus, M: Starlikeness properties of a new integral operator for meromorphic functions. J Appl Math 2011, 8 (2011). Article, ID 804150

24. Goyal, SP, Prajapat, JK: A new class of meromorphic multivalent functions involving certain linear operator. Tamsui Oxford, J Math Sci. 25(2), 167-176 (2009)

doi:10.1186/1029-242X-2011-121

Cite this article as: Mohammed and Darus: Integral operators on new families of meromorphic functions of complex order. Journal of Inequalities and Applications 2011 2011:121.

\section{Submit your manuscript to a SpringerOpen ${ }^{\circ}$ journal and benefit from:}

Convenient online submission

- Rigorous peer review

- Immediate publication on acceptance

- Open access: articles freely available online

- High visibility within the field

- Retaining the copyright to your article

Submit your next manuscript at $\gg$ springeropen.com 